\title{
Haemophilus parainfluenzae as a marker of the upper respiratory tract microbiota changes under the influence of preoperative prophylaxis with or without postoperative treatment in patients with lung cancer
}

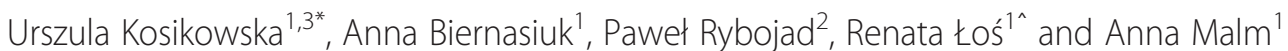

\begin{abstract}
Background: Haemophili are representative microbiota of the upper respiratory tract. The aim of this study was to assess the effects of perioperative antimicrobial prophylaxis and/or postoperative treatment on Haemophilus parainfluenzae prevalence, and antimicrobial sensitivity in short-term hospitalized patients with lung cancer who underwent surgery.

Results: Samples were collected from 30 short-term hospitalized patients with lung cancer and from 65 healthy people. The nasal and throat specimens were taken twice from each patient: before (El, Examination I), on the fourth/fifth day (Ell, Examination II) after surgery, and once from healthy people. The isolates identification and antimicrobial susceptibility were detected by routine diagnostic methods. H. parainfluenzae was found in throat specimens of 42/65 (64.6\%) healthy people, while in 19/30 (63.3\%) lung cancer patients in El $(p=0.6203)$ and in $13 / 30(43.3 \%)$ ones in Ell $(p=0.0106)$. Neither the disease itself nor short-term hospitalization with perioperative prophylaxis alone affected $\mathrm{H}$. parainfluenzae prevalence in Ell, while perioperative prophylaxis with postoperative treatment significantly decreased its colonization in Ell. The differences in the number of patients colonized by Candida spp. in El and in Ell were observed ( $p=0.0082)$.Totally, 23/58 (39.7 \%) of H. parainfluenzae isolates were resistant mainly to beta-lactams; among 11 ampicillin-resistant isolates only 3 were beta-lactamase positive.
\end{abstract}

Conclusions: The antimicrobial perioperative prophylaxis together with postoperative treatment may disturb the composition of the airways microbiota represented by $\mathrm{H}$. parainfluenzae, in addition to selecting the resistant strains of bacteria and promoting yeasts colonization in lung cancer patients undergoing surgery.

Keywords: Haemophilus parainfluenzae, Candida spp., Respiratory microbiota, Lung cancer, Perioperative prophylaxis, Postoperative treatment

\footnotetext{
* Correspondence: urszula.kosikowska@umlub.pl

Deceased

'Department of Pharmaceutical Microbiology with Laboratory for

Microbiological Diagnostics, Medical University of Lublin, Lublin, Poland

${ }^{3}$ Department of Pharmaceutical Microbiology with Laboratory for

Microbiological Diagnostics, Medical University of Lublin, Chodzki Str. 1,

Lublin 20-093, Poland

Full list of author information is available at the end of the article
} 


\section{Background}

The microbiota is very important in the life style conditions and health safety [1-3]. Among numerous microorganisms of the upper respiratory tract microbiota, Haemophilus spp. may be common and representative. Haemophili play a role in preventing the establishment of potential pathogens and are very important for the proper functioning of the human body, including defense mechanisms $[4,5]$. The changes in the composition of microbiota can cause various dysfunctions of the protective barrier of the airways and may contribute to an increase in mucosal colonization by pathogenic microorganisms including the environmental ones, eg hospital microflora [6-8]. Additionally, it can be regarded as a reservoir of opportunistic pathogens. The microbiota disturbance may predispose to other bacteria or fungi colonization and respiratory infections [6, 9]. According to some studies, fungi like Candida spp. or Aspergillus spp., Gram-negative rods (mainly Enterobacteriaceae and Pseudomonadaceae) and Gram-positive bacteria (eg Staphylococcus spp. or Streptococcus spp.) are the important etiological factors of diseases in patients with lung cancer $[9,10]$.

Haemophili, mainly Haemophilus influenzae and occasionally Haemophilus parainfluenzae, can cause a variety of invasive, chronic or recurrent diseases $[2,11,12] . H$. parainfluenzae is increasingly recognized as an opportunistic pathogen responsible for various infections [13, 14], including the respiratory tract infections [15-17], endocarditis [18-20], bacteraemia or even sepsis $[21,22]$. Qualitative and quantitative changes in resident or transient members of the respiratory tract microbiota, eg Haemophilus spp., may be a risk factor of endogenous infections, which are an important medical problem among several groups of patients, eg immunocompromised patients.

Surgery remains the most effective treatment in lung cancer [23]. As patients with neoplastic changes already have the weakened defense mechanisms, prophylactic antibiotic treatment is usually administrated before, during, or after diagnostic and therapeutic procedures [24-27]. During hospitalization, both the patient's microbiota and its susceptibility to antimicrobials may undergo qualitative and quantitative changes. It depends on the environmental conditions and the type (prophylaxis and/or antimicrobial treatment) as well as the length of an antibiotic application.

The present study seeks to determine the prevalence and antimicrobial sensitivity of the haemophili species (mainly $H$. parainfluenzae) as the upper respiratory tract microbiota, depending on the perioperative prophylaxis and/or postoperative treatment with commonly used antibiotics in short-term hospitalized lung cancer patients who underwent surgery.

\section{Methods}

\section{Patients}

Thirty patients aged 43-75 years old (average 62.1) with lung cancer who were admitted to the Department of Thoracic Surgery of Medical University of Lublin (between February 2011 and March 2012) were included in the study. Lung cancer was determined before surgery on the basis of an examination of specimens obtained during bronchoscopy, thin needle biopsy or sputum cytology. At the beginning of the sampling, none of the patients qualified for the study had any clinical evidence of viral or bacterial airways infections (normal body temperature and leukocyte count). Within 30 days before being admitted to hospital, no patients had taken any antimicrobial agents or drugs influencing the immunological system. Moreover, they neither had any blood transfusions nor suffered from an allergic disease. All patients with resectable lung cancer were subjected to microbiological studies in a comparable time both before surgery, which was performed on the day of hospital admission and before antibiotic treatment (EI, Examination I), and four or five days after the surgery accompanied by preoperative antimicrobial prophylaxis with or without postoperative antibiotic treatment (EII, Examination II). All patients were operated on one or two days after hospital admission. Each patient received preoperative antimicrobial prophylaxis with beta-lactams (cefuroxime or cefazolin) with or without amikacin. For prophylactic purposes the commonest route of administration was one single dose of antibiotics; the drug should be given not later than about 0.5 hour before the commencement of surgery; in prolonged surgery redosing at 4 hour's intervals is indicated. Moreover, 15 patients had beta-lactams treatment and 1 patient had beta-lactams with amikacin treatment extended onto the postoperative period for the next four or five days.

The control (reference) group was established out of 65 healthy volunteers aged 19-75 years old (average 45.3 years). All patients agreed to participate in the research. The study was approved by the Ethical Committee of Medical University of Lublin (No. KE-0254/75/2011).

\section{Microbiological assay}

The specimens - one swab from throat and two swabs from nose (from the left and right nostrils independently) - were taken from each lung cancer patient twice: first on the day of hospital admission and before antibiotic treatment (EI) and then on the fourth/fifth day after thoracic surgery (EII). A total of 180 specimens were taken from 30 patients with lung cancer including 60 swabs from the throat (in EI - 30, and in EII - 30) and 120 nasal swabs (in EI - 60, and in EII - 60). Additionally, 65 swabs were taken from healthy people's throat once. 
The specimens were taken by means of sterile cotton swabs and they were immediately placed onto the appropriate nonselective medium (5\% sheep blood agar) and the selective medium for haemophili (Haemophilus chocolate agar, HAEM, bioMérieux, France), Gram-negative rods (McConkey agar, Oxoid, England), and for fungi (Sabouraud dextrose agar, bioMérieux, France; BBL Chromagar Candida, Becton Dickinson and Company, USA).

The blood agar, McConkey and Sabouraud medium were incubated under aerobic conditions for 18-48 hours at $35{ }^{\circ} \mathrm{C}$. After incubation, colonies from both McConkey agar and Sabouraud dextrose agar were selected and cultured on the agar media for bacteria and for fungi for identification of the isolates. The presence of bacteria and yeasts in the upper airways in at least one sample was considered as colonization. The strains of Gram-negative bacteria and fungi were identified using biochemical microtests (bioMérieux, France) API 20E (for Enterobacteriaceae family) and API 20NE (for Pseudomonadaceae family) or API ID 32C (for Candida spp.). The ability of Candida strains to produce hyphae, pseudohyphae or chlamydospores was also evaluated.

The HAEM medium for haemophili was incubated in the atmosphere with an increased $5 \% \mathrm{CO}_{2}$ concentration (appropriate for microaerophilic bacteria) for 18-48 hours at $35{ }^{\circ} \mathrm{C}$. After incubation, the growth of bacteria in the form of individual colonies or from abundant to a very abundant number of morphologically different colonies on Chocolate agar was observed. For the initial identification of isolated haemophili, morphological characteristics of the colonies growing on HAEM agar and the requirements for hemin (X factor) and nicotinamide adenine dinucleotide (V factor) on TSA (Tripticasein Soy Lab-Agar, Biocorp, Poland) medium with diagnostic discs DD3 (X factor), DD4 (V factor), DD5 (both X and V factors) obtained from Oxoid (England) were determined. Biochemical identification of isolates was carried out using the API NH microtest (bioMérieux, France). The haemophili isolates were differentiated based on various observable properties in the growth morphology (e.g. the shape and size of the colony, smooth or rough surface, texture, colony elevation), on a set of biochemical reactions (according to API NH results) and antimicrobial susceptibility results.

Antibiotic sensitivities of $H$. parainfluenzae isolates from patients were determined by the disc diffusion method using Haemophilus Test Medium (HTM, Oxoid, England) according to the Clinical Laboratory Standards Institute (CLSI) recommendation for Haemophilus species [28]. Direct colony suspensions standardized to 0.5 McFarland standard $\left(\sim 10^{8}\right.$ CFU, colony forming units $/ \mathrm{ml}$ ) were prepared using the colonies from an overnight HAEM agar incubation at $35{ }^{\circ} \mathrm{C}$ in the atmosphere with about $5 \% \mathrm{CO}_{2} . H$. influenzae ATCC10211 was used to verify the growth promotion properties of HTM. Different discs with antimicrobial agents (BD BBL, Becton Dickinson and Company, USA), namely ampicillin $(10 \mu \mathrm{g})$, amoxicillin-clavulanic acid $(20 / 10 \mu \mathrm{g})$, ampicillin-sulbactam $(10 / 10 \mu \mathrm{g})$, cefazoline $(30 \mu \mathrm{g})$, cefuroxime $(30 \mu \mathrm{g})$, cefotaxime $(30 \mu \mathrm{g})$, ceftazidime $(30 \mu \mathrm{g})$, imipenem $(10 \mu \mathrm{g})$, aztreoname $(30 \mu \mathrm{g})$, azithromycin $(15 \mu \mathrm{g})$, tetracycline $(30 \mu \mathrm{g})$, trimethoprim/ sulfamethoxazole $(1.25 / 23.75 \mu \mathrm{g})$, ciprofloxacin $(5 \mu \mathrm{g})$ were used. Multidrug resistant haemophili isolates were defined as having resistance to at least three different classes of antimicrobials. Isolates resistant to ampicillin were screened for beta $\beta$-lactamase production using Pen test (API NH, bioMerieux, France) and the nitrocefin as chromogenic cephalosporin method (Cefinase disks, BD BBL, Becton Dickinson and Company, USA). The test was considered positive if the colour changed from yellow to purple for cephalosporin.

\section{Statistical analysis}

Data processing and analysis were performed using StatSoft, Inc. Statistica 2010 for Windows. Contingency table analysis for comparing proportions was done by Fisher's exact test. The relative risk (RR) and its $95 \%$ confidence intervals (CI) were calculated. Statistical significance was established at $p<0.05$.

\section{Results}

In $30 / 30$ (100 \%) patients with lung cancer cefuroxime or cefazolin was used during preoperative antimicrobial prophylaxis; in 14/30 (46.7\%) cases it was combined with amikacin. Perioperative prophylaxis alone was applied to $14 / 30$ (46.7 \%) patients. Preoperative prophylaxis with postoperative treatment was applied in 16/30 $(53.3 \%)$ cases - 15/30 (50 \%) patients underwent cefuroxime or cefazolin and 1/30 (3.3\%) patient had betalactams with amikacin treatment.

Haemophili were cultured in 21/60 samples taken from the throat and in $0 / 120$ samples taken from the nasal specimens of patients with lung cancer during examinations EI and EII. According to data presented in Table 1, the prevalence of throat colonization by $H$. parainfluenzae in lung cancer patients was higher in EI (19/30, 63.3 \%) compared to EII (13/30, $43.3 \%)$. These differences were not statistically significant $(p=0.1954)$. Totally, 20/30 (66.7\%) patients were colonized by $H$. parainfluenzae in both examinations (EI + EII). Besides, $H$. influenzae was isolated from $1 / 30$ (3.3 \%) patient (only in EI).

Haemophili were cultured in 49/65 (75.4\%) samples taken from the throat of healthy people. Among 49/65 healthy people colonized by haemophili, 42/65 (64.6\%) 
Table 1 Frequency of the throat colonization by Haemophilus parainfluenzae in healthy people and in patients with lung cancer before (Examination I, El) and after (Examination II, Ell) perioperative prophylaxis without and with postoperative treatment

\begin{tabular}{|c|c|c|c|c|}
\hline \multirow[t]{2}{*}{ Group of patients } & \multicolumn{4}{|l|}{ No. (\%) of people } \\
\hline & Uncolonized by haemophili & Colonized by Haemophilus parainfluenzae & RR $(95 \% \mathrm{Cl})$ & $\mathrm{p}$ value \\
\hline Healthy people $(n=65)$ & $16(24.6)$ & $42(64.6)$ & Referent & \\
\hline \multicolumn{5}{|c|}{ Patients with lung cancer $(n=30)$} \\
\hline El & $10(33.3)$ & $19(63.3)$ & $0.8(0.4-1.5)$ & 0.6203 \\
\hline Ell & $17(56.7)$ & $13(43.3)$ & $0.5(0.3-0.8)$ & 0.0106 \\
\hline $\mathrm{El}+\mathrm{Ell}$ & $10(33.3)$ & $20(66.7)$ & $0.8(0.4-1.6)$ & 0.6264 \\
\hline
\end{tabular}

Abbreviations: $R R$ relative risk

cases were colonized by $H$. parainfluenzae, 1/65 (1.5\%) by $H$. influenzae and 6/65 (9.2\%) by other Haemophilus spp. Statistically significant differences between the number of healthy people and patients with lung cancer colonized by haemophili were detected in EII $(49 / 65$ vs. $13 / 30, p=0.0048)$, but not in EI (49/65 vs.20/30, $p=0.4591$ ). As was shown in Table 1 , statistically significant differences between the number of healthy people and patients with lung cancer colonized by $H$. parainfluenzae were also detected in EII $(p=0.0106)$, but not in EI $(p=0.6203)$.

Detail analysis of lung cancer patients colonized by $H$. parainfluenzae revealed that $12 / 30$ (40 \%) patients were colonized both in EI and EII, 7/30 (23.3 \%) only in EI, while $1 / 30$ (3.3\%) patient only in EII (Fig. 1). Among 20 patients colonized totally by $H$. parainfluenzae, in $10 / 20$ (50\%) patients cefazolin or cefuroxime were used only as perioperative prophylaxis, while in 9/20 (45\%) patients cefuroxime and in $1 / 20(5 \%)$ patient cefuroxime and amikacin were applied both as preoperative prophylaxis and as postoperative treatment.

Considering the total number of patients with antimicrobial perioperative prophylaxis alone $(14 / 30$,

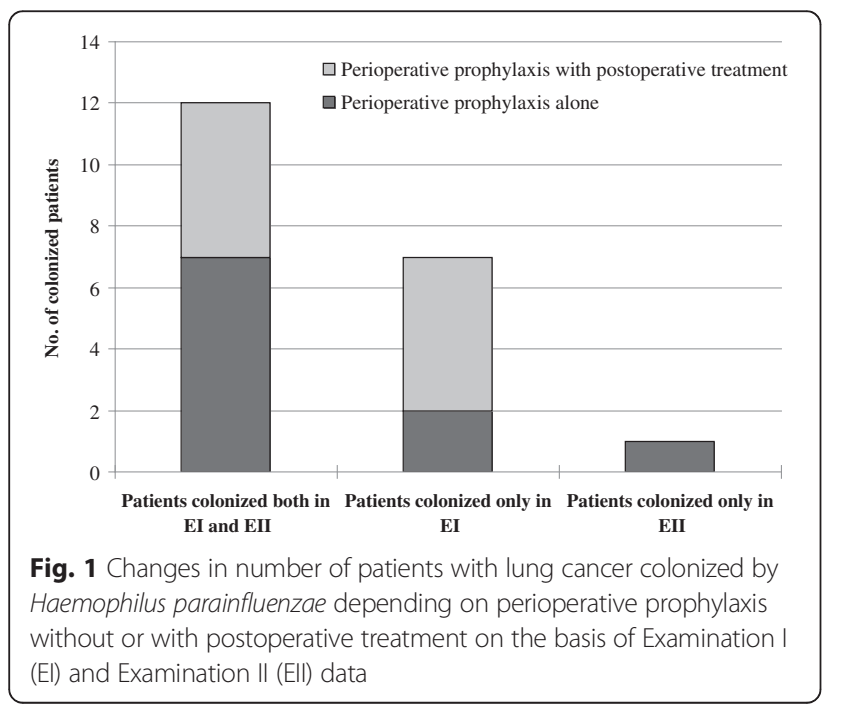

$46.7 \%)$ or with antimicrobial perioperative prophylaxis together with postoperative treatment (16/30, $53.3 \%)$ and the number of patients colonized by $H$. parainfluenzae in EI and EII in both groups of patients (Fig. 1), it was shown that perioperative prophylaxis alone did not significantly affected $H$. parainfluenzae prevalence $(p=0.7477)$, while perioperative prophylaxis with postoperative treatment significantly decreased the number of colonized patients $(p=0.0626)$. Moreover, statistically significant differences in $H$. parainfluenzae colonization between healthy people and patients with lung cancer undergoing perioperative prophylaxis with postoperative treatment were also found in EII $(p=0.023)$, but not in patients with perioperative prophylaxis alone $(p=0.7609)$.

According to Table 2, the prevalence of throat colonization by Candida spp., mainly $C$. albicans, in lung cancer patients was higher in EII (23/30, $76.7 \%$ ) compared to EI $(12 / 30,40 \%)$. C. albicans was isolated from $9 / 30(30 \%)$ and from 16/30 (53.3 \%) patients in EI and EII, respectively. Additionally, 5 of C. famata (EI - 1, EII - 4), 4 of C. glabrata (EI- 2, EII - 2), and 1 of C. krusei (EI- 0, EII - 1) strains were isolated. The differences in the number of patients colonized by Candida spp. in EI and in EII were observed $(p=0.0082)$. Additionally, among patients with lung cancer single isolates of Gram-negative rods were cultured (Escherichia coli, Klebsiella pneumoniae, Morganella morganii, Aeromonas hydrophila, Alcaligenes xylosoxidans, Acinetobacter baumannii, Kluyvera spp) mainly in EII.

On the basis of growth morphology, biochemical properties and profile of antimicrobial resistance differences among the number of phenotypically various $H$. parainfluenzae isolates selected from one patient before and after surgery were determined. Totally, $58 \mathrm{H}$. parainfluenzae isolates were cultured from 20 patients with lung cancer - 32/58 (55.2 \%) from 19/20 (90 \%) patients before (EI) and 26/58 (44.8 \%) isolates from 13/20 (65\%) patients after (EII) surgery. Among all selected isolates, $23 / 58$ (39.7 \%) were resistant to different antimicrobials - 13/58 (22.4 \%) in EI, and 10/58 (17.3\%) in 
Table 2 Prevalence of throat colonization by Candida spp. and other than Haemophilus spp.Gram-negative bacteria in patients with lung cancer before (Examination I, EI) and after (Examination II, Ell) perioperative prophylaxis without and with postoperative treatment

\begin{tabular}{|c|c|c|c|c|}
\hline \multirow{2}{*}{$\begin{array}{l}\text { No. of } \\
\text { patient }\end{array}$} & \multicolumn{2}{|l|}{ El } & \multicolumn{2}{|l|}{ E II } \\
\hline & Yeasts & Gram-negative bacteria & Yeasts & Gram-negative bacteria \\
\hline 2 & Candida albicans & Klyuvera spp. & Candida albicans & Klyuvera spp. \\
\hline 3 & Candida albicans & - & Candida albicans & - \\
\hline 5 & - & - & Candida albicans & - \\
\hline 6 & - & - & Candida albicans & - \\
\hline 7 & - & - & Candida famata & - \\
\hline 8 & - & Aeromonas hydrophila & Candida albicans & - \\
\hline 9 & - & - & Candida albicans & - \\
\hline 11 & - & - & Candida albicans & Morganella morganii \\
\hline 12 & Candida albicans & - & Candida albicans & - \\
\hline 13 & - & - & Candida krusei & Alcaligenes xylosoxidans \\
\hline 14 & - & - & Candida famata & - \\
\hline 15 & Candida albicans & - & Candida albicans & - \\
\hline 16 & Candida albicans & - & Candida albicans & - \\
\hline 17 & Candida glabrata & - & Candida glabrata & Klebsiella pneumoniae Escherichia coli \\
\hline 20 & - & Escherichia coli & - & - \\
\hline 21 & Candida albicans & - & Candida albicans $^{a}$ & - \\
\hline 22 & Candida albicans & - & Candida albicans & - \\
\hline 23 & Candida albicans & - & Candida albicans & Acinetobacter baumanii \\
\hline 24 & Candida albicans & - & Candida albicans & - \\
\hline 25 & & - & Candida albicans & - \\
\hline 26 & Candida glabrata Candida famata & - & Candida glabrata Candida famata & - \\
\hline 27 & - & - & - & Klebsiella pneumoniae \\
\hline 29 & - & - & Candida albicans & - \\
\hline 30 & - & - & Candida famata & - \\
\hline
\end{tabular}

${ }^{a}$ Two phenotypically different strains of Candida albicans were selected

EII ( 9 isolates in patients without and 1 isolate in a patient with prolonged treatment with antimicrobials). Totally, $13 / 58(22.4 \%)$ isolates of $H$. parainfluenzae were resistant to beta-lactams. In the group of patients with preoperative prophylaxis alone, 6/58 (10.3\%) H. parainfluenzae isolates both in EI and EII were resistant mainly to beta-lactams (Table 3). Two phenotypically different isolates of $H$. parainfluenzae with increased resistance to antimicrobials were selected in EII only from one patient with cefuroxime as prophylaxis. Among 11/58 (19 \%) ampicillin-resistant isolates only three were ampicillinresistant beta-lactamase positive. Among them 2 isolates were selected both in EI and EII from one patient and therefore it was assumed to be the same strain. Besides, 9 other beta-lactamase negative isolates were resistant to ampicillin and beta-lactams co-administered with a betalactamases inhibitor - clavulanic acid or sulbactam. Multidrug resistance (MDR) was detected in 1 isolate, which was resistant to beta-lactams (AmAmcAtm), macrolides (Azm) and trimethoprim/sulphametoxazole (Sxt).

\section{Discussion}

It is known that in most cases very strong cancer treatments used today may often affect the health condition and change the defense mechanisms, microbiota conditions and the immune system [29-31]. This treatment, either alone or in combination, usually kills cancer cells and also damages the immune system cells and it can increase the risk of infections or pathogens and opportunistic microbials colonization.

According to our results, the prevalence of haemophili, especially $H$. parainfluenzae, in the airways of investigated patients was relatively stable after a shortterm perioperative prophylaxis. This may suggest that perioperative prophylaxis used does not significantly interfere with the microbiota. Conversely, perioperative 
Table 3 The effect of antimicrobial preoperative prophylaxis with or without postoperative treatment on the resistance of Haemophilus parainfluenzae isolates selected from patients with lung cancer in Examination I (El) and in Examination II (EII)

\begin{tabular}{|c|c|c|c|c|c|c|}
\hline \multicolumn{3}{|c|}{ Colonized patients } & \multicolumn{4}{|c|}{ Haemophilus parainfluenzae isolates $(n=58)$} \\
\hline \multirow{2}{*}{$\begin{array}{l}\text { Number of } \\
\text { patients }\end{array}$} & \multirow[t]{2}{*}{ Prophylaxis } & \multirow[t]{2}{*}{ Treatment } & \multirow[t]{2}{*}{ Examination } & \multicolumn{2}{|c|}{ No. (\%) of isolates } & \multirow{2}{*}{$\begin{array}{l}\text { Profile of resistance } \\
\text { (No. of isolates) }\end{array}$} \\
\hline & & & & Sensitive & $\overline{\text { Resistant }}$ & \\
\hline \multirow[t]{17}{*}{10} & Yes & No & $\mathrm{El}(n=18)$ & $9(15.5)$ & $9(15.5)$ & Ampen+ $(1)$ \\
\hline & & & & & & Te (1) \\
\hline & & & & & & Sxt (1) \\
\hline & & & & & & SxtTe (1) \\
\hline & & & & & & $\mathrm{CazCtxCz}(2)$ \\
\hline & & & & & & AmAmcSam (1) \\
\hline & & & & & & AmAmcCazAtmTe (1) \\
\hline & & & & & & AmAmcSamCtxCzAtm (1) \\
\hline & & & Ell $(n=16)$ & $7(12.1)$ & $9(15.5)$ & $A m_{\text {Pen+ }}(1)$ \\
\hline & & & & & & $\mathrm{Cz}(1)$ \\
\hline & & & & & & Te (1) \\
\hline & & & & & & Sxt (1) \\
\hline & & & & & & SxtTe (1) \\
\hline & & & & & & AmAmcSam (1) \\
\hline & & & & & & AmAmcSamCtxCzAtm (1) \\
\hline & & & & & & $\mathrm{Am}_{\mathrm{Pen}+} \mathrm{SamCazCzCxmCtx}$ (1) \\
\hline & & & & & & AmAmcSamCazCtxCzAtmSxt (1) \\
\hline \multirow[t]{5}{*}{10} & Yes & Yes & $\mathrm{El}(n=14)$ & $10(17.2)$ & $4(6.9)$ & $\mathrm{Cz}(1)$ \\
\hline & & & & & & Sxt (1) \\
\hline & & & & & & AmAmcAtmAzmSxt (1) \\
\hline & & & & & & AmSamCazCzlpm (1) \\
\hline & & & Ell $(n=10)$ & $9(15.5)$ & $1(1.7)$ & Sxt (1) \\
\hline
\end{tabular}

Abbreviations: Am ampicillin; AmC amoksicillin/clavulanic acid; An amikacin; Atm aztreonam; Azm azithromycin; Caz ceftazidime; Ctx cefotaxime; Cxm cefuroxime; Cz cefazoline; Ipm imipenem; Sxt trimethoprim/sulfametoksazol; Te tetracycline; Pen + penicyllinase-positive

prophylaxis and postoperative treatment with antibiotics may contribute to both qualitative and quantitative changes within the microbiota represented by $H$. parainfluenzae in our studies. However, it is difficult to define clearly the factors influencing elimination of these bacteria from the airways of patients with lung cancer. In our opinion, the disturbed microbiota composition promotes the mucosa colonization both by the fungi and other bacteria. This was demonstrated mainly in the case of Candida spp., but also in Gram-negative bacteria belonging to different species. It could also be the reason for the occurrence of ampicillin-resistant and other beta-lactams resistant isolates or multidrug resistant bacteria during our studies. According to literature, changes in airways colonization in patients with lung cancer after antimicrobial treatment were observed both in nasopharynx samples by Gram-negative rods [32] and in throat samples by Candida strains [33]. This may be a precursor to bacterial or fungal respiratory tract infections, which are favoured by, for example, lung cancer and treatment or surgical procedures. Drakulovic et al [34] showed that hospital microflora may colonize patients in the first week of hospitalization and this colonization may increase the severity of the underlying disease and the process of healing after surgery.

Up to $80 \%$ of healthy individuals may carry strains belonging to the genus Haemophilus creating nonpathogenic microbiota in the upper respiratory tract [16]. H. parainfluenzae is a typical commensal of the indigenous microbiota with unclear pathogenicity in contrast to the accepted pathogenicity of $H$. influenzae [11, 35-37]. These species occasionally, especially when the host's immune system is suppressed, may be a cause of localized or even systemic infections [16, 35-38]. Hofstra and co-workers [39] observed the increase of $H$. parainfluenzae in all volunteers during experimental human Rhinovirus acute infection. This increase was significant $(p=0.0098)$ but reversible and returned to the baseline level after the infection was cleared. This fact may indicate an 
important role of these bacteria in the proper functioning of the non-specific immune defence of macroorganisms against colonization by pathogens.

One of undesirable side effects of antibacterial treatment is the deficiency in the nonspecific immune system and colonization of e.g. the mucous membranes with some pathogenic or opportunistic microorganisms [11, 29, 40-43]. However, bacteriological diagnosis depends on the identification of species or their characteristics because the state of microbiota condition is still rare in many laboratories due to the nutritional requirements of these bacteria, the cost and special skills involved.

The effect of both perioperative prophylaxis and a prolonged use of antibiotics on microflora depends on many factors, related both to the type of antimicrobials and to the properties of the microorganisms [27, 40, 44-46]. Beta-lactams, especially cephalosporins, are appropriate first line antimicrobials for most surgical procedures [27, 45]. Perioperative prophylaxis or postsurgical treatment can reduce or even eliminate transient or resident flora and it may be very important as prevention against endogenous infections [25, 41, 42, 47]. It has to be underlined though that they do not protect against pathogens' colonization. Additionally, in some people this may lead to increased susceptibility to pathogen's colonization or to opportunistic infections.

This is also compatible with the findings of other authors who noted that disruption of normal microflora may predispose people to infection $[8,26]$. Kager et al. [45] investigated microflora in faecal samples of patients who underwent colorectal surgery and received antimicrobial prophylaxis and a prolonged tinidazole administration period, enterococci and streptococci decreased and the number of anaerobic bacteria increased. The authors noted the occurrence of postoperative infections with $E$. coli etiology.

Beta-lactams are the most widely used antibiotics, and beta-lactamases are a greatest source of resistance to them [48]. The phenotypically expressed resistance to beta-lactam antibiotics in haemophili is dependent mainly on the level of production of beta-lactamases and the presence of penicillin-binding protein (PBP) with lowered affinity for these antibiotics as a target site [49-51]. Beta-lactamases were detected in $H$. parainfluenzae isolates from both healthy people and patients with respiratory tract infections. Uraz et al. [52] showed the presence of about $57 \%$ beta-lactamase positive species among the throat cultures of children with upper respiratory tract infections. In literature there is rare information about beta-lactamases and of altered penicillin-binding proteins in $H$. parainfluenzae [51, 53]. According to Gromkova et al. [54, 55], DNA transformation probably plays a major role in the spread of drug resistance in $H$. parainfluenzae. It seems that especially efficient in transformation were the cells classified as biotypes II and I, which are the prevalent biotypes in the world with an ability to develop competence. During natural transformation the transfer of genes occurs via free DNA (from dead or lysed cells) from the surrounding medium by competent bacterial cells $[56,57]$. The capacity of absorption of the extracellular DNA by transformation may explain the acquisition of resistance or resistance gene exchange with other bacteria.

\section{Conclusions}

Our results for the first time confirm that antimicrobials prophylaxis together with prolonged postoperative treatment with antimicrobials during short-term hospitalization may disturb the airways microbiota using $H$. parainfluen$z a e$ as a marker in lung cancer patients who underwent thoracic surgery. In contrast, neither the disease itself nor short-term hospitalization with perioperative prophylaxis alone significantly affected $H$. parainfluenzae prevalence. In our opinion, haemophili are common and representative bacteria within the upper respiratory tract, and they are a very simple marker of microbiota condition with regard to their role as a protective factor for the host organism. Additionally, a study of the respiratory microbiota composition and its disturbance may unfold new insights and approaches to the pathogenesis of lung diseases.

\section{Ethics approval}

The Ethics Committee of the Medical University of Lublin approved study protocol (KE-0254/75/2011).

\section{Consent statement}

All participants, including the healthy volunteers, gave a conscious oral consent to take part in the studies.

\section{Availability of data and materials}

Data presented in this study are complete. No supplementary files are attached.

\section{Abbreviations \\ El: Examination I; Ell: Examination II; HAEM: Haemophilus chocolate agar; HTM: Haemophilus test medium; TSA: Tripticasein Soy Lab-Agar.}

\section{Competing interests}

The authors declare that they have no competing interests.

\section{Author's contribution}

UK conceived, designed and coordinated the study, collected haemophili specimens, analysed the data and drafted the manuscript; $A B$ collected fung specimens; PR collected patients' protocols and the nasopharynx and sputum specimens; Rt $^{\dagger}$ collected Gram-negative specimens; AM analysed the data and critically reviewed the manuscript. All co-authors read and approved the final manuscript. 


\section{Acknowledgements}

This research was done during a project supported by Medical University in Lublin.

\section{Funding}

None.

\section{Author details}

${ }^{1}$ Department of Pharmaceutical Microbiology with Laboratory for Microbiological Diagnostics, Medical University of Lublin, Lublin, Poland. ${ }^{2}$ Department of Thoracic Surgery, Medical University of Lublin, Lublin, Poland. ${ }^{3}$ Department of Pharmaceutical Microbiology with Laboratory for Microbiological Diagnostics, Medical University of Lublin, Chodzki Str. 1, Lublin 20-093, Poland.

\section{Received: 26 August 2015 Accepted: 29 March 2016 Published online: 06 April 2016}

\section{References}

1. Hui AW, Lau HW, Chan TH, Tsui SK. The human microbiota: a new direction in the investigation of thoracic diseases. J Thorac Dis. 2013;5 Suppl 2:127-31.

2. Purcell P, Jary H, Perry A, Perry JD, Stewart CJ, Nelson A, et al. Polymicrobial airway bacterial communities in adult bronchiectasis patients. BMC Microbiol. 2014;14:130. doi:10.1186/1471-2180-14-130.

3. Ivanov II, Littman DR. Modulation of immune homeostasis by commensal bacteria. Curr Opin Microbiol. 2011;14:106-14.

4. Dickson RP, Huang YJ, Martinez FJ, Huffnagle GB. The lung microbiome and viral-induced exacerbations of chronic obstructive pulmonary disease: new observations, novel approaches. Am J Respir Crit Care Med. 2013:188:1185-6.

5. Nørskov-Lauritsen N. Classification, identification, and clinical significance of Haemophilus and Aggregatibacter species with host specificity for humans. Clin Microbiol Rev. 2014;27(2):214-40.

6. Berghmans T, Sculier JP, Klastersky J. A prospective study of infections in lung cancer patients admitted to the hospital. Chest. 2003;124:114-20.

7. Cardenas PA, Cooper PJ, Cox MJ, Chico M, Arias C, Moffatt MF, Cookson WM. Upper airways microbiota in antibiotic-naïve wheezing and healthy infants from the tropics of Rural Ecuador. PLoS One. 2012;7, e46803.

8. Cui Z, Zhou Y, Li H, Zhang Y, Zhang S, Tang S, Guo X. Complex sputum microbial composition in patients with pulmonary tuberculosis. BMC Microbiol. 2012;12:276. doi:10.1186/1471-2180-12-276.

9. Lanoix JP, Pluquet E, Lescure FX, Bentayeb H, Lecuyer E, Boutemy $M$, et al. Bacterial infection profiles in lung cancer patients with febrile neutropenia. BMC Infect Dis. 2011;11:183.

10. Akinosoglou KS, Karkoulias K, Marangos M. Infectious complications in patients with lung cancer. Eur Rev Med Pharmacol Sci. 2013;17:8-18.

11. Albritton WL. Infections due to Haemophilus species other than $\mathrm{H}$. influenzae. Annu Rev Microbiol. 1982;11:199-216.

12. Tufvesson E, Bjermer L, Ekberg M. Patients with chronic obstructive pulmonary disease and chronically colonized with Haemophilus influenzae during stable disease phase have increased airway inflammation. Int J Chron Obstruct Pulmon Dis. 2015:10:881-9.

13. Cardines R, Giufre M, Ciofi degli Atti ML, Accogli M, Mastrantonio P, Cerquetti M. Haemophilus parainfluenzae meningitis in an adult associated with acute otitis media. New Microbiol. 2009;32:213-5.

14. Gardenier JC, Sawyer RG, Sifri CD, Brayman K, Wispelway B, Bonatti H. Peritonitis caused by Haemophilus parainfluenzae, Leifsonia aquatica, and Gordonia spp. in a patient undergoing continuous ambulatory peritoneal dialysis. Surg Infect (Larchmt). 2012;13:409-12.

15. Hill SL, Mitchell JL, Stockley RA, Wilson R. The role of Haemophilus parainfluenzae in COPD. Chest. 2000;117:293S.

16. Middleton AM, Dowling RB, Mitchell JL, Watanabe S, Rutman A, Pritchard K, et al. Haemophilus parainfluenzae infection of respiratory mucosa. Resp Med. 2003;97:375-81.

17. Rhind GB, Gould GA, Ahmad F, Croughan MJ, Calder MA. Haemophilus influenzae and $H$. parainfluenzae infections: comparison of clinical features. Br Med J. 1985:291:707-8.

18. Darras-Joly C, Lortholary O, Mainardi JL, Etienne J, Guillevin L, Acar J. Haemophilus endocarditis: report of 42 cases in adults and review. Haemophilus Endocarditis Study Group. Clin Infect Dis. 1997;24:1087-94.
19. Yew HS, Chambers ST, Roberts SA, Holland DJ, Julian KA, Raymond NJ, et al. Association between HACEK bacteraemia and endocarditis. J Med Microbiol. 2014;63:892-5

20. Nwaohiri N, Urban C. Tricuspid valve endocarditis caused by Haemophilus parainfluenzae: a case report and review of the literature. Diagn Microbiol Infect Dis. 2009;64:216-9.

21. Bruun B, Christensen JJ, Kilian M. Bacteriemia caused by a beta-lactamase producing $H$. parainfluenzae strain of a new biotype. Acta Pathol Microbiol Immunol Scand B. 1984;92:135-8.

22. Govind B, Veeraraghavan B, Anandan S, Thomas N. Haemophilus parainfluenzae: report of an unusual cause of neonatal sepsis and a literature review. J Infect Dev Ctries. 2012;6:748-50.

23. American Cancer Society. The history of cancer. Accessed at: http://www. cancer.org/acs/groups/cid/documents/webcontent/002048-pdf.pdf

24. Ma Y, Bao L, Wang H, Shi S. A multi-center study on the prophylactic application of antibiotics in aseptic operations. Genet Mol Res. 2015;14: 2356-64.

25. Sullivan $\AA$, Edlund C, Nord CE. Effect of antimicrobial agents on the ecological balance of human microflora. Lancet Infect Dis. 2001;1:101-14.

26. Seppälä $\mathrm{H}, \mathrm{Al}$-Juhaish $\mathrm{M}$, Järvinen $\mathrm{H}$, Laitinen $\mathrm{R}$, Huovinen P. Effect of prophylactic antibiotics on antimicrobial resistance of viridans streptococci in the normal flora of cataract surgery patients. J Cataract Refract Surg. 2004;30:307-15.

27. SIGN Guideline No 104. Antibiotic prophylaxis in surgery. A national clinical guideline. Scottish Intercollegiate Guidelines Network. Edinburgh 2008. Accessed at: www.sign.ac.uk/pdf/sign104.pdf.

28. Clinical and Laboratory Standards Institute (CLSI). Performance standards for antimicrobial susceptibility testing; Twenty-fourth informational supplement M100-S22. Clinical and Laboratory Standards Institute, Wayne, PA 2012.

29. American Cancer Society. Infections in people with cancer. 2015. Accessed at: http://www.cancer.org/acs/groups/cid/documents/webcontent/002871pdf.pdf

30. Dickson RP, Erb-Downward JR, Huffnagle GB. The role of the bacterial microbiome in lung disease. Expert Rev Respir Med. 2013:7:245-57.

31. Zanussi S, Serraino D, Dolcetti R, Berretta M, De Paoli P. Cancer, aging and immune reconstitution. Anticancer Agents Med Chem. 2013:13:1310-24.

32. Los R, Rybojad P, Gozdziuk K, Malm A. Dynamics of nasopharyngeal colonization by gram-negative rods in patients with resectable lung cancer during short-term hospitalization. New Microbiol. 2008;31:507-12.

33. Biernasiuk A, Korona-Głowniak I, Mahorowska-Kiciak I, Rybojad P, Malm A. Pharyngeal Candida sp. strains in patients with non-small cell lung cancer. Mikol Lek. 2006;13:89-93.

34. Drakulovic MB, Bauer TT, Torres A, Gonzalez J, Rodriguez M-J, Angrill J. Initial bacterial colonization in patients admitted to a Respiratory Intensive Care Unit: bacteriological pattern and risk factors. Respiration. 2001;68:58-66.

35. Choi D, Thermidor M, Cunha BA. Haemophilus parainfluenzae mitral prosthetic valve endocarditis in an intravenous drug abuser. Heart Lung. 2005;34:152-4.

36. Frankard J, Rodriguez-Villalobos H, Struelens MJ, Jacobs F. Haemophilus parainfluenzae: an undiagnosed pathogen of biliary tract infections? Eur J Clin Microbiol Infect Dis. 2004:23:46-8.

37. Mitchell $\mathrm{J}$, Hill SL. Immune response to Haemophilus parainfluenzae in patients with chronic obstructive lung disease. Clin Diagn Lab Immunol. 2000:7:25-30.

38. Pillai A, Mitchell $J$, Hill SL, Stockley RA. A case of Haemophilus parainfluenzae pneumonia. Thorax. 2000:55:623-4

39. Hofstra JJ, Matamoros S, van de Pol MA, de Wever B, Tanck MW, WendtKnol H, Deijs M, van der Hoek L, Wolthers KC, Molenkamp R, Visser CE, Sterk PJ, Lutter R, de Jong MD. Changes in microbiota during experimental human Rhinovirus infection. BMC Infect Dis. 2015;15:336. doi:10.1186/s12879015-1081-y.

40. Malm A, Korona-Glowniak I, Kasprzycka A, Rybojad P, Górniewski G, Furmanik F. Impact of preoperative chemotherapy and/or antimicrobial prophylaxis on dynamics of nasopharyngeal microflora in patients with lung cancer during short-term hospitalization. Bull Vet Inst Pulawy. 2002:46(Suppl):167-70.

41. Lundberg C. Postoperative infections in the upper respiratory tract. Scand J Infect Dis Suppl. 1988;57:35-40.

42. Rybojad P, Los R, Sawicki M, Tabarkiewicz J, Malm A. Anaerobic bacteria colonizing the lower airways in lung cancer patients. Folia Histochem Cytobiol. 2011;49:263-6.

43. Stoutenbeek CP, van Saene HK, Miranda DR, Zandstra DF. The effect of selective decontamination of the digestive tract on colonization and 
infection rate in multiple trauma patients. Intensive Care Med. 1984;10:185-92.

44. Kager L, Ljungdahl I, Malmborg AS, Nord CE. Effect of tinidazole prophylaxis on the normal microflora in patients undergoing colorectal surgery. Scand J Infect Dis Suppl. 1981;26:84-91.

45. Kager L, Ljungdahl I, Malmborg AS, Nord CE, Pieper R, Dahlgren P. Antibiotic prophylaxis with cefoxitin in colorectal surgery: effect on the colon microflora and septic complications - a clinical model for prediction of the benefit and risks in using a new antibiotic in prophylaxis. Ann Surg. 1981;193:277-82.

46. Bratzler DW, Dellinger EP, Olsen KM, Perl TM, Auwaerter PG, Bolon MK, Fish DN, Napolitano LM, Sawyer RG, Slain D, Steinberg JP, Weinstein RA. Clinical practice guidelines for antimicrobial prophylaxis in surgery. Am J Health Syst Pharm. 2013;70(3):195-283.

47. Karaman E, Alimoglu Y, Aygun G, Kilic E, Yagiz C. Effect of septoplasty and per-operative antibiotic prophylaxis on nasal flora. B-ENT. 2012;8:13-9.

48. Livermorea DM, Brown DFJ. Detection of $\beta$-lactamase-mediated resistance. J Antimicrob Chemother. 2001;48 Suppl 1:59-64.

49. Gilbert DN, Moellering RC, Eliopoulos GM, Sande MA. The Sanford Guide to Antimicrobial Therapy. 33rd ed. Hyde Park, VT: Antimicrobial Therapy; 2003. p. 24-7.

50. James PA, Reeves DS. Bacterial resistance to cephalosporins as a function of outer membrane permeability and access to their target. J Chemother. 1996;8 Suppl 2:37-47.

51. Tristram SG, Pitout MJ, Forward K, Campbell S, Nichols S, Davidson RJ. Characterization of extended-spectrum beta-lactamase-producing isolates of Haemophilus parainfluenzae. J Antimicrob Chemother. 2008;61:509-14.

52. Uraz G, Simşek H, Celik B. Beta-lactamase activities and resistance to antibiotics of Haemophilus influenzae, H. parainfluenzae and H. aphrophilus strains identified in throat cultures from children. Drug Metabol Drug Interact. 2000;16:217-28

53. Takahata S, Ida T, Senju N, Sanbongi Y, Miyata A, Maebashi K, Hoshiko S. Horizontal gene transfer of ftsl, the gene encoding penicillin binding protein 3 in Haemophilus influenzae. Antimicrob Agents Chemother. 2007:51:1589-95.

54. Gromkova RC, Goodgal S. Transformation by plasmid and chromosomal DNAs in Haemophilus parainfluenzae. Biochem Biophys Res Commun. 1979;88:1428-34

55. Gromkova RC, Mottalini TC, Dove MG. Genetic transformation in Haemophilus parainfluenzae clinical isolates. Curr Microbiol. 1998;37:123-6.

56. Lorenz MG, Wackernagel W. Bacterial gene transfer by natural genetic transformation in the environment. Microbiol Rev. 1994;58:563-602.

57. Redfield RJ, Findlay WA, Bosse J, Kroll JS, Cameron AD, Nash JH. Evolution of competence and DNA uptake specificity in the Pasteurellaceae. BMC Evol Biol. 2006;6:82

\section{Submit your next manuscript to BioMed Central and we will help you at every step:}

- We accept pre-submission inquiries

- Our selector tool helps you to find the most relevant journal

- We provide round the clock customer support

- Convenient online submission

- Thorough peer review

- Inclusion in PubMed and all major indexing services

- Maximum visibility for your research

Submit your manuscript at www biomedcentral.com/submit

) Biomed Central 